山્પ FRANÇAISE

$>\mathrm{DE}$

필 PÉDAGOGIE
Revue française de pédagogie

Recherches en éducation

199 | avril-mai-juin 2017

L'histoire des disciplines : un champ de recherche en mutation

\title{
VEILLARD Laurent. La formation professionnelle initiale. Apprendre dans l'alternance entre différents contextes
}

Rennes : Presses universitaires de Rennes, 2017, 308 p.

\section{Laurent Filliettaz}

\section{(2) OpenEdition}

Journals

Édition électronique

URL : https://journals.openedition.org/rfp/6765

DOI : 10.4000/rfp.6765

ISSN : 2105-2913

Éditeur

ENS Éditions

Édition imprimée

Date de publication : 30 juin 2017

Pagination : 153-154

ISBN : 979-10-362-0108-0

ISSN : 0556-7807

Référence électronique

Laurent Filliettaz, «VEILLARD Laurent. La formation professionnelle initiale. Apprendre dans l'alternance entre différents contextes », Revue française de pédagogie [En ligne], 199 | avril-mai-juin 2017, mis en ligne le 30 juin 2017, consulté le 08 janvier 2022. URL : http://journals.openedition.org/rfp/6765 ; DOI : https://doi.org/10.4000/rfp.6765

Ce document a été généré automatiquement le 8 janvier 2022.

(c) tous droits réservés 


\title{
VEILLARD Laurent. La formation professionnelle initiale. Apprendre dans l'alternance entre différents contextes
}

\author{
Rennes : Presses universitaires de Rennes, 2017, 308 p.
}

\section{Laurent Filliettaz}

\section{RÉFÉRENCE}

VEILLARD Laurent. La formation professionnelle initiale. Apprendre dans l'alternance entre différents contextes. Rennes : Presses universitaires de Rennes, 2017, 308 p.

1 Dans le contexte francophone, les ouvrages portant sur les problématiques de formation professionnelle initiale et qui abordent ce champ dans ses différentes facettes, historiques, didactiques et en lien avec la problématique de l'alternance, sont fort peu nombreux. Pendant longtemps en effet, la recherche en éducation a clairement délaissé la formation professionnelle initiale, un secteur éducatif pourtant porteur d'innombrables enjeux sur le plan socio-économique.

De ce point de vue, l'ouvrage publié par Laurent Veillard et conçu à partir de sa thèse d'habilitation à diriger des recherches présente une grande originalité. Il propose, de manière synthétique et remarquablement informée, une somme importante de connaissances qui se sont constituées dans des champs disciplinaires variés, sans pour autant donner lieu à une présentation intégrée. C'est là tout l'intérêt d'un ouvrage qui combine les apports de la didactique des disciplines, de la didactique de la formation professionnelle, de la sociologie de la formation, ou encore de la psychologie culturelle des apprentissages pour montrer comment, dans le contexte français plus particulièrement, les jeunes apprennent des métiers au carrefour de plusieurs espaces éducatifs et productifs.

3 Le livre ne manque pas de cibler un éventail élargi de lecteurs, chercheurs ou formateurs, intéressés à mieux connaître les développements récents de la recherche 
portant sur l'alternance en formation professionnelle, un domaine qui s'est considérablement consolidé et transformé récemment pour répondre à des enjeux à la fois politiques et éducatifs de première importance. Comme le rappelle l'auteur dans l'introduction, le monde du travail connait d'innombrables accélérations et changements techniques. Les échanges économiques se mondialisent, les savoirs et les savoir-faire sont sans cesse renouvelés, de nouveaux métiers apparaissent pendant que d'autres s'éclipsent. Ces transformations toujours plus rapides des sphères productives posent des problèmes considérables pour organiser la formation initiale et préparer les jeunes générations au monde du travail. Et pourtant, ces changements prennent forme dans un terrain éminemment sédimenté et façonné par des forces construites par l'histoire. Ils ne peuvent être ni compris ni affrontés sans mesurer pleinement les arrangements institutionnels complexes impliqués dans les systèmes et les pratiques de la formation professionnelle initiale.

4 La problématique de la formation professionnelle initiale ne se réduit pas à la question des politiques éducatives et des dispositifs. Elle impose de comprendre également comment ces dispositifs sont investis par les acteurs sur le terrain. Ceci concerne d'abord les enseignants et les formateurs, et leur capacité de mise en œuvre des référentiels et des programmes. Mais ceci concerne aussi le point de vue des apprenants eux-mêmes. Comment vivent-ils ces situations de formation? Quels apprentissages sont-ils en mesure d'effectuer dans les différents lieux qu'ils fréquentent dans le cours de leur formation? Et de quel poids pèsent les origines sociales et les parcours scolaires antérieurs sur les modalités d'engagement et les apprentissages?

5 On le voit, les questions posées par cet ouvrage sont nombreuses et portent sur des enjeux de diverses natures. Pour y répondre, Laurent Veillard propose un panorama des études réalisées au niveau international dans différents champs disciplinaires, et qui suivent néanmoins une ligne directrice aussi claire que convaincante. Cette idée structurante est fondée sur l'hypothèse selon laquelle serait à l'œuvre un processus de division croissante du travail à l'intérieur même des institutions spécialisées dans la préparation des jeunes au monde professionnel. Laurent Veillard observe en effet que pratiquement tous les dispositifs de formation professionnelle initiale actuels, quels que soient les pays ou les domaines professionnels, combinent différents contextes de transmission et d'apprentissage, animés chacun par des acteurs spécialisés. Dans cette perspective, l'ouvrage a pour ambition de comprendre à la fois les origines historiques de ce processus de division du travail, son évolution récente, ses traductions dans les structures actuelles de formation, ses conséquences sur l'activité des formateurs et finalement ses effets sur les apprentissages.

6 Attestant une démarche fortement pluridisciplinaire, l'ouvrage permet de penser les continuités entre une analyse de l'activité des formateurs et une étude des trajectoires d'apprenants. Il s'intéresse également aux processus de transposition et d'appropriation de savoirs et à une analyse des modes d'engagement des acteurs dans l'action. Enfin, l'ouvrage propose une analyse des données à des granularités variables, allant du large empan à des analyses plus microscopiques. Sur le plan des méthodes de recherche en sciences de l'éducation, l'ouvrage procède à un inventaire très maitrisé des enjeux qui caractérisent la recherche en formation professionnelle et ouvre des voies prometteuses pour des recherches à venir. 
$7 \quad$ Une autre qualité de l'ouvrage est la perspective historique qu'il permet d'adopter sur des problématiques de didactique de la formation professionnelle. Comme le montre remarquablement le premier chapitre, des enjeux de pouvoir viennent interférer dans les questions de formation et de division du travail, tout comme dans les pratiques de référence et les objets de savoirs au cœur des pratiques de transmission. Ce détour par l'histoire permet de porter un regard à la fois profond et renouvelé sur les enjeux et les débats actuels de l'alternance dans la formation professionnelle. On saluera également le chapitre 2, consacré à l'examen de travaux sociologiques sur les évolutions récentes des structures des systèmes de formation professionnelle initiale, ainsi qu'aux publics concernés. Ce chapitre présente une excellente synthèse de la dynamique de ces évolutions. Les chapitres 3,4 et 5 se complètent réciproquement et abordent la problématique des apprentissages dans chacun des lieux de l'alternance: les écoles professionnelles; la place de travail ; et enfin les dispositifs d'alternance qui cherchent à combiner des situations en écoles avec des périodes de formation en situation de travail. À ce propos, le chapitre 4 présente l'intérêt de faire référence à l'abondante littérature produite récemment dans le champ anglo-saxon du Workplace Learning. Enfin, le sixième et dernier chapitre propose une définition élargie de l'alternance, qui permet de mieux prendre en considération les problèmes posés par les multiples transitions que doivent accomplir les apprenants entre différents contextes d'apprentissage. À la lumière d'études portant sur la question du transfert et sur celle des transitions socio-culturelles, le chapitre montre en quoi l'éclatement des formations en de multiples composantes didactiques pose des difficultés aux apprenants, qui se trouvent souvent face à la nécessité de construire eux-mêmes les liens entre des connaissances et des expériences éparses.

8 Dans son ensemble, l'ouvrage produit par Laurent Veillard témoigne d'un très gros travail de lecture et de synthèse, mettant à la disposition de la communauté scientifique une somme de connaissances originales et non disponibles à ce jour sous la forme d'une monographie. L'ouvrage présente un bel équilibre entre une présentation d'un champ de recherche pluridisciplinaire, un diagnostic de quelques difficultés didactiques inhérentes à des dispositifs de formation professionnelle en alternance et des pistes d'ingénierie permettant de dépasser ces difficultés. C'est ainsi un champ de pratique et de recherche qui s'en trouve consolidé et projeté vers des travaux futurs dans ce domaine.

\section{AUTEURS}

\section{LAURENT FILLIETTAZ}

Université de Genève 